\title{
Deep genome sequencing and variation analysis of 13 inbred mouse strains defines candidate phenotypic alleles, private variation and homozygous truncating mutations
}

\author{
Anthony G. Doran ${ }^{1}$, Kim Wong ${ }^{1}$, Jonathan Flint ${ }^{2}$, David J. Adams ${ }^{1}$, Kent W. Hunter ${ }^{3^{*}}$ and Thomas M. Keane ${ }^{1 *}$
}

\begin{abstract}
Background: The Mouse Genomes Project is an ongoing collaborative effort to sequence the genomes of the common laboratory mouse strains. In 2011, the initial analysis of sequence variation across 17 strains found $56.7 \mathrm{M}$ unique single nucleotide polymorphisms (SNPs) and 8.8 M indels. We carry out deep sequencing of 13 additional inbred strains (BUB/BnJ, C57BL/10J, C57BR/CdJ, C58/J, DBA/1J, I/LnJ, KK/HiJ, MOLF/EiJ, NZB/B1NJ, NZW/LacJ, RF/J, $\mathrm{SEA} / \mathrm{GnJ}$ and ST/bJ), cataloguing molecular variation within and across the strains. These strains include important models for immune response, leukaemia, age-related hearing loss and rheumatoid arthritis. We now have several examples of fully sequenced closely related strains that are divergent for several disease phenotypes.

Results: Approximately 27.4 M unique SNPs and $5 \mathrm{M}$ indels are identified across these strains compared to the C57BL/6 J reference genome (GRCm38). The amount of variation found in the inbred laboratory mouse genome has increased to $71 \mathrm{M} \mathrm{SNPs}$ and $12 \mathrm{M}$ indels. We investigate the genetic basis of highly penetrant cancer susceptibility in RF/J finding private novel missense mutations in DNA damage repair and highly cancer associated genes. We use two highly related strains (DBA/1J and DBA/2J) to investigate the genetic basis of collagen-induced arthritis susceptibility.

Conclusions: This paper significantly expands the catalogue of fully sequenced laboratory mouse strains and now contains several examples of highly genetically similar strains with divergent phenotypes. We show how studying private missense mutations can lead to insights into the genetic mechanism for a highly penetrant phenotype.
\end{abstract}

Keywords: Sequencing, Mouse genomes, Laboratory mouse, Genomic variation, Disease, Cancer, arthritis, Biological pathways

\section{Background}

Genetic mutations (such as single nucleotide polymorphisms (SNPs), indels and structural variants (SVs)) are commonly involved in the dysregulation of normal gene function and frequently the cause of human diseases. Laboratory mouse strains are one of the primary model organisms used to study human disease and have

\footnotetext{
* Correspondence: hunterk@mail.nih.gov; tk2@sanger.ac.uk

${ }^{3}$ Laboratory of Cancer Biology and Genetics, $\mathrm{NCl}, \mathrm{NIH}$, Bethesda, Maryland, USA

'Wellcome Trust Sanger Institute, Hinxton, Cambridge CB10 1HH, UK

Full list of author information is available at the end of the article
}

excelled as a model system in which to identify and characterise the role that genomic variation plays in susceptibility and pathogenesis $[1,2]$. A multitude of inbred laboratory strains are now commonly employed in the examination of a wide range of human diseases [3].

The first draft version of the mouse reference genome was produced based on a whole genome shotgun sequencing strategy of a female C57BL/6J strain mouse [4] followed several years later by the finished genome sequence [5]. The mouse reference genome enabled a whole set of new applications and technologies such as 
many new genetic screens [6], the creation of null alleles for the majority of genes [7], and accelerated the discovery of genetic diversity in other laboratory mouse strains [8]. The Mouse Genomes Project (MGP) was initiated with the primary goals to sequence the genomes of the common laboratory mouse strains, catalogue molecular variation and produce full chromosome sequences of each strain [9]. The initial phase of the MGP focused on sequencing and variation analysis of a representative subset of the most commonly used inbred mouse strains. This culminated in the identification of $57.7 \mathrm{M}$ SNPs, 8.8 $\mathrm{M}$ indels and $0.71 \mathrm{M} \mathrm{SVs}$ across the genomes of 17 of the most commonly used strains [9].

Here, we present a significant expansion of the MGP variation catalogue by deep sequencing $(30-60 \times)$ and variation analysis of 13 strains: $\mathrm{BUB} / \mathrm{BnJ}, \mathrm{C} 57 \mathrm{BL} / 10 \mathrm{~J}$, C57BR/cdJ, C58/J, DBA/1J, I/LnJ, KK/HiJ, MOLF/EiJ, NZB/B1NJ, NZW/LacJ, RF/J, SEA/GnJ and ST/bJ. These represent a diverse set of phenotypes and genotypes of inbred mouse strains that are commonly used to study rheumatoid arthritis (RA) (DBA/1J), diabetes $(\mathrm{KK} / \mathrm{HiJ})$, immunity (C57BL/10J, NZB/B1NJ, NZW/LacJ), cancer (C58/J, RF/J), development (I/LnJ, SEA/GnJ) and agerelated diseases (BUB/BnJ, C57BR/cdJ, MOLF/EiJ, ST/ $\mathrm{bJ})$. The catalogue of variants derived from these strains has been incorporated into the current MGP variation catalogue to determine private and shared variation among the 13 strains and refine private variation identification across all strains in the catalogue.

We also demonstrate how variants in these 13 strains can be utilised to investigate the genetic basis of disease. In the first analysis, predicted damaging SNPs private to the $R F / J$ strain were used to identify candidate genes and biological pathways involved in tumorigenesis and disease progression. The RF/J strain is an important model in cancer studies due to a naturally high predisposition to several cancers including acute myeloid leukaemia and thymic lymphoma [10,11]. The second analysis involved the identification of candidate genes for collagen-induced arthritis (CIA) susceptibility, a model of human RA, by comparison of SNPs between two genetically related strains with divergent phenotypes. Candidate genes identified in the analysis of these two strains, DBA/1J (susceptible) and DBA/2J (resistant), were then further investigated to identify potential causal pathways and biological processes controlling pathogenesis of this disease.

\section{Results and discussion}

\section{A phenotypically diverse set of laboratory mouse strains} A total of 13 genetically and phenotypically diverse inbred laboratory mouse strains were chosen for analysis in the current study (Fig. 1). These strains were selected from across the inbred mouse subspecies groups and represent a large number of model systems used to study human disease. This dataset includes strains that are genetically similar to the current MGP strains, such as DBA/1J and C57BL/10J, as well as strains that are genetically divergent, such as the wild-derived Japanese strain MOLF/EiJ (Fig. 1).

With this expansion, there are now several examples of fully sequenced strains which are closely related to other strains in the catalogue but are divergent for disease phenotypes. For example, C57BR/cdJ mice are closely related to the mouse reference genome strain $\mathrm{C} 57 \mathrm{BL} / 6$, but display different patterns of learning behaviour [12]. Following avoidance training, C57BL/6 mice exhibit poor conditioned avoidance performance, whereas C57BR/cd] perform much better in avoidance tests [13]. Another example of genetically similar but phenotypically divergent strains include DBA/1J and DBA/2J which are susceptible and resistant, respectively, to CIA. DBA/1J mice are commonly used as a model for RA and exhibit several similarities to the human disease including synovitis and degradation of the bone and cartilage tissue [14].

Among this expansion of the variation catalogue are several strains with similar phenotypes such as the NZB/ B1NJ and NZW/LacJ strains. These strains are widely used as models for autoimmune abnormalities. For example, both strains develop anti-DNA antibodies. Also, F1 hybrids of these two strains develop an autoimmune disease resembling human systemic lupus erythematosus [15].

Another interesting addition to the variation catalogue examined here is the RF/J strain $[10,11]$. This strain is an important model for cancer research due to a naturally high incidence rate of acute myeloid leukaemia and tumour development. This strain also exhibits a high spontaneous rate of glomerular hyalinisation and glomerulosclerosis [16]. Each of the remaining strains also exhibit several notable phenotypes; $\mathrm{BUB} / \mathrm{BnJ}$ are homozygous for the Mass $f^{\text {frings }}$ allele, which is responsible for early onset (3-4 weeks of age) hearing impairment [17]; a high proportion of $\mathrm{I} / \mathrm{LnJ}$ mice display agenesis of the corpus callosum which is associated with developmental delays and impaired motor function in humans [18]; SEA/GnJ mice segregate for the short ear allele $\left(B M P 5^{\text {se }}\right)$, which causes numerous defects in cartilage and bone tissue [19]; both the ST/bJ and MOLF/EiJ strains are homozygous for the retinal degeneration allele $P d e 6 b^{r d 1}$ [20] (11853768); and $\mathrm{C} 58 / \mathrm{J}$ and $\mathrm{KK} / \mathrm{HiJ}$ mice are homozygous for the age-related hearing loss mutation $C d h 23^{a h l}$ [21].

\section{Identification of SNPs and indels}

The 13 mouse strains were sequenced to a mapped depth of $30-60 \times$ on the Illumina HiSeq platform at a read length of $101 \mathrm{bp}$ paired-end (Table 1). In total, over $1.8 \mathrm{~Tb}$ of raw sequencing data were generated. All raw sequencing reads are available from the European 


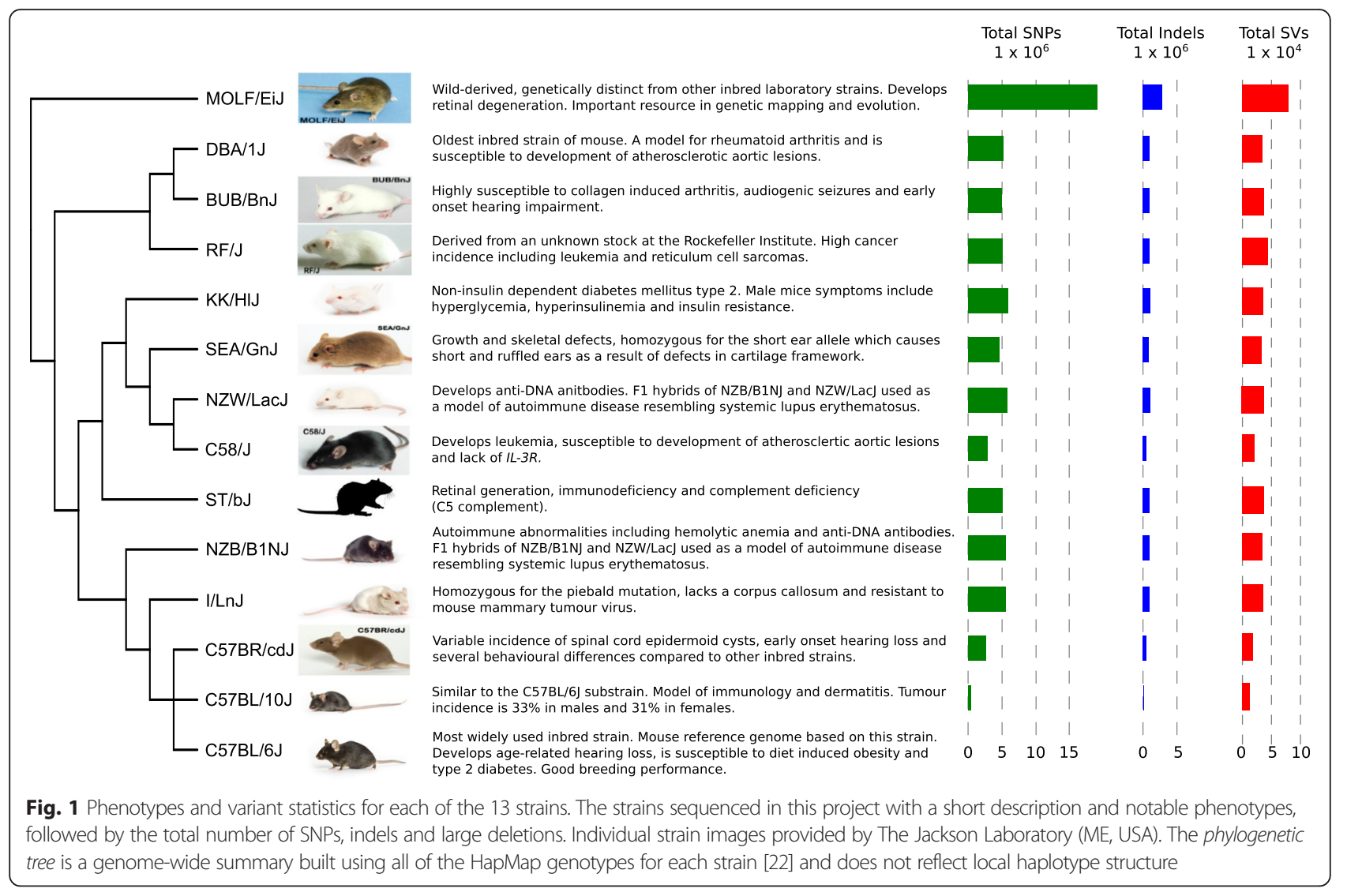

Nucleotide Archive (ERP000927; see Table S1 in Additional file 1). Sequencing reads were aligned to the GRCm38 mouse reference genome using BWA-mem, followed by a local realignment around indels using GATK. The aligned sequence reads are also available from the MGP (ftp://ftp-mouse.sanger.ac.uk).
A total of 27.39 M high confidence homozygous SNPs were identified across the genomes of 13 inbred laboratory mouse strains (Fig. 1). This catalogue of variants, when compared to the MGP variation catalogue of 23 strains, contains $2.78 \mathrm{M}(10.15 \%)$ novel variant sites, of which $2.33 \mathrm{M}(8.51 \%)$ are private to one of the 13

Table 1 Sequencing, alignment and variant statistics

\begin{tabular}{lccllllll}
\hline Strain & Raw bases (Gb) & Mapped coverage & SNPs & Private & Indels & Private & SVs & Private \\
\hline BUB/BnJ & 135.42 & 38.92 & $4,982,043$ & 54,418 & 929,617 & 19,187 & 37,034 & 5375 \\
C57BL/10J & 108.43 & 29.85 & 349,702 & 2733 & 83,930 & 1893 & 12,939 & 9904 \\
C57BR/cdJ & 140.94 & 39.76 & $2,601,138$ & 9683 & 485,803 & 6307 & 18,927 & 3462 \\
C58/J & 153.06 & 43.76 & $2,834,841$ & 27,805 & 547,151 & 11,726 & 20,666 & 3777 \\
DBA/1J & 139.79 & 38.55 & $5,129,920$ & 9001 & 955,621 & 9684 & 34,239 & 4480 \\
I/LnJ & 124.97 & 34.11 & $5,547,267$ & 78,583 & $1,013,686$ & 24,628 & 36,612 & 6342 \\
KK/HiJ & 151.60 & 44.42 & $5,893,784$ & 94,110 & $1,078,555$ & 31,084 & 36,588 & 3725 \\
MOLF/EiJ & 112.55 & 30.68 & $19,027,669$ & $1,818,029$ & $2,892,638$ & 367,208 & 79,239 & 17,394 \\
NZB/B1NJ & 133.24 & 36.15 & $5,473,276$ & 54,189 & $1,014,782$ & 20,670 & 34,406 & 3164 \\
NZW/LacJ & 160.46 & 49.06 & $5,736,044$ & 52,902 & $1,051,170$ & 21,397 & 38,207 & 3308 \\
RF/J & 147.33 & 43.18 & $5,090,115$ & 32,390 & 937,091 & 13,440 & 44,205 & 11,685 \\
SEA/GnJ & 134.23 & 38.67 & $4,603,720$ & 30,263 & 857,599 & 12,729 & 33,033 & 3686 \\
ST/Bj & 223.17 & 63.74 & $5,107,777$ & 70,064 & 978,238 & 24,325 & 37,350 & 4474 \\
\hline
\end{tabular}

Total sequencing in Gigabases (Gb), mapped coverage (based on 3Gb genome) and the total and private number of SNPs, indels and large deletions is shown 
strains in this study when compared to the entire variation catalogue (Fig. 2; Additional file 2). The wildderived MOLF/EiJ strain contained the most individual variation both in terms of total $(19.03 \mathrm{M})$ and private SNPs $(1.82 \mathrm{M})$. Conversely, the C57BL/10J strain, which is genetically much more similar to the reference genome, displayed the least amount of total and private variation (349,702 and 2733). The total number of SNPs and short indels shared between each pair of strains contained in the MGP variation catalogue is given in Additional file 3 .

In total $4.81 \mathrm{M}$ high quality homozygous indels were identified, $0.64 \mathrm{M}$ of which are novel indels sites and $0.56 \mathrm{M}$ of these are private to one of the 13 strains when compared to the all other strains included in the MGP variation catalogue (Fig. 2; Additional file 2). The size of the $2.91 \mathrm{M}$ deletions identified is in the range of 1-46 bp and the size of $2.5 \mathrm{M}$ insertions is in the range of 1-29 bp. Similar to the number of SNPs identified, MOLF/EiJ contained the largest number of short indels relative to the reference genome $(2.89 \mathrm{M}$ total and 367,208 private). Additional comparisons of the total number of indels shared between each pair of strains is contained in Additional file 3.

To ensure the accuracy of our SNP calling pipeline, genotypes from the mouse HapMap [22] for approximately 138,000 locations were obtained and compared to the corresponding genotypes in each of the 13 strains involved in this study. After removing missing genotypes and mapping genotype locations from NCBIm37 to GRCm38 of the reference genome, between 115,572 (MOLF/EiJ) and 129,825 (C58/J) genotypes remained for comparison with each strain. Sensitivity of our calling pipeline was determined by assessing the concordance rate for each of the individual strains. Greater than $98 \%$ of genotypes were concordant for each of the individual strains (Additional file 4). In fact, a total of $1.64 \mathrm{M}$ genotypes were compared, in which $1.62 \mathrm{M}$ $(98.68 \%)$ were concordant. All of the variants in this study were incorporated into the MGP v5 variation catalogue and are available from the project website (ftp://ftp-mouse.sanger.ac.uk) and the European Variation Archive (PRJEB11471). The MGP v5 catalogue comprises 36 inbred laboratory strains with 70.72 M SNP sites, $39.23 \mathrm{M}$ of which are private to an individual strain, and $11.29 \mathrm{M}$ short indels, $6 \mathrm{M}$ of which are private.

\section{Functional consequences of SNPs and indels}

The Variant Effect Predictor (VEP) tool [23] was used to assign functional annotations to the SNP and indel variants across the 13 strains (Table 2 and Additional file 5). The majority of SNPs are found in intergenic (46.58-52.28 \% across strains; $50.57 \%$ of all SNPs identified) and intronic (15.74-18.58\%; $18.08 \%$ ) regions.
Additionally, a large amount of variation was identified upand downstream of protein-coding genes (3.91-4.47 \%; $3.93 \%$ and $3.79-4.43 \%$; $4.05 \%$, respectively), and within the 3' $(0.20-0.24 \%)$ and $5^{\prime}(0.02-0.04 \%)$ untranslated regions (UTR).

Although most SNPs were found in non-coding regions, a large amount of variation was located in protein-coding regions (76,108 SNPs). Of particular note are SNPs that affect the protein-coding sequence, such as splice variants and non-synonymous SNPs. A total of 27,416 (0.1 \%) missense SNPs were identified across the 13 strains. MOLF/EiJ contained the largest number of missense variants $(17,311)$; however, only 2288 of these were private to this strain. One particular novel missense mutation is a $\mathrm{G} \rightarrow \mathrm{A}$ transition in exon 4 of the Pctp gene identified in the NZW/LacJ and NZB/BlNJ strains. This mutation, at position $89,987,348$ on chromosome 11 , is also shared with the NZO/HlLtJ strain which suggests fixation of the mutation in progenitor mice prior to development of the NZ inbred mouse strains [24]. This mutation causes an arginine to histidine change at amino acid position 120, which leads to inactivation of the protein. Although the physiological implications of inactivating Pctp are not yet fully understood, inactivation has been linked to changes in control of hepatic cholesterol homeostasis [25] and impaired biliary lipid secretion [26].

We identified 178 SNPs that result in a stop gain or loss. MOLF/EiJ contained the most individual variation in coding regions including 23 stop losses and 85 stop gains; however only one stop loss and 18 stop gain variants were private to this strain. C57BL/10J was the only strain in which a stop gain or loss was not identified. Several mouse strains are known to carry homozygous mutations that lead to the introduction of a premature stop in the coding sequence. Among the list of stop gains identified in our study was a $\mathrm{C} \rightarrow \mathrm{A}$ mutation which causes a premature stop codon in the Pde6b gene on chromosome 5 at position 108,421,265. This mutation is found in several strains included in the MGP variation catalogue, including three from this study: BUB/BnJ, MOLF/EiJ and ST/bJ. This mutation, rs49995481, results in truncation of the predicted protein in which over half of the protein is missing and is known to cause early onset severe retinal degeneration [27].

To determine the impact of an amino acid substitution, SIFT (Sorting Intolerant from Tolerant) [28] and grantham matrix scores (GMS) [29] were estimated for all non-synonymous SNPs. SIFT scores $\leq 0.05$ are usually considered to be indicative of a deleterious mutation. Although most non-synonymous SNPs are tolerated, 11,182 in 6241 genes were predicted to have a deleterious effect. Similar to SIFT, GMS enables the identification of moderately radical $(100<$ GMS $\leq 150)$ and radical 
Table 2 Predicted consequences of variants

\begin{tabular}{|c|c|c|c|c|c|c|c|}
\hline \multirow[b]{2}{*}{ Strain } & \multicolumn{3}{|l|}{ SNPS } & \multicolumn{2}{|l|}{ Indels } & \multicolumn{2}{|l|}{ Exonic SVs } \\
\hline & Synonymous & Non-synonymous & Stop gain/loss & Frameshift variant & Inframe variant & Deletions & Insertions \\
\hline $\mathrm{B} \cup \mathrm{B} / \mathrm{BnJ}$ & 9751 & 6050 & 44 & 41 & 169 & 1247 & 720 \\
\hline C57BL/10J & 581 & 298 & 0 & 4 & 8 & 57 & 102 \\
\hline C57BR/cdJ & 4400 & 2948 & 18 & 26 & 52 & 288 & 227 \\
\hline C58/J & 5238 & 3362 & 23 & 35 & 69 & 330 & 254 \\
\hline $\mathrm{DBA} / 1 \mathrm{~J}$ & 9782 & 6198 & 39 & 53 & 149 & 419 & 389 \\
\hline I/Ln」 & 10389 & 6249 & 42 & 52 & 150 & 690 & 1671 \\
\hline KK/HiJ & 10816 & 6197 & 50 & 56 & 140 & 599 & 462 \\
\hline MOLF/EiJ & 33710 & 17,311 & 108 & 172 & 439 & 897 & 1305 \\
\hline NZB/B1NJ & 10403 & 6367 & 43 & 62 & 146 & 500 & 390 \\
\hline NZW/LacJ & 10434 & 6326 & 46 & 61 & 146 & 673 & 425 \\
\hline $\mathrm{RF} / \mathrm{J}$ & 9891 & 6174 & 39 & 56 & 140 & 2033 & 862 \\
\hline SEA/GnJ & 9309 & 6027 & 38 & 57 & 138 & 1284 & 614 \\
\hline ST/bJ & 9021 & 5578 & 45 & 59 & 133 & 713 & 413 \\
\hline
\end{tabular}

(GMS > 150) non-synonymous substitutions. Only 3337 (10.78\%) of non-synonymous substitutions are considered radical using GMS, although 1130 of these are also annotated as deleterious using SIFT.

Similar to SNPs, most indels are intergenic (47.76\%), intronic $(18.82 \%)$ or within $5 \mathrm{~kb}$ upstream (4.26\%) or downstream (4.27 \%) of a coding gene. Although, in general, indels found in intergenic or intronic regions are not known to have an effect on protein-coding genes, several strains carry a 5 bp deletion at the branching point of intron 7 of the Il3ra gene which leads to skipping of exon 8 [30]. This mutation, on chromosome 14 at position $14,350,904$, is found in nine MGP strains including five from this study: BUB/BnJ, C58/J, I/LnJ, $\mathrm{NZB} / \mathrm{BINJ}$ and RF/J. A small number of indels cause an in-frame deletion (368) or insertion (278) and only 308 indels cause a frameshift. As with missense SNPs, MOLF/EiJ contained the largest number of frameshift mutations (172); however, proportional to the total number of indels identified in this strain, this number was approximately the same as the other strains (0.0006\%). Several strains are known to carry indels that affect the protein-coding function of genes, such as a known TA deletion in the haemolytic complement gene. This deletion, at position $35,043,207$ on chromosome 2, leads to the creation of a premature stop codon 4 bases after the deletion [31] and consequently macrophages from mice carrying this mutation fail to secrete complement 5 (C5) [32]. This mutation is carried by several strains in the MGP catalogue, including six strains from this study: I/LnJ, KK/HiJ, MOLF/EiJ, NZB/B1NJ, RF/J and ST/bJ. Mice deficient for $\mathrm{C} 5$ have previously demonstrated enhanced protection against development of severe glaucoma compared to mice of the same strain sufficient for C5 [33].

\section{Novel and private variation}

To identify variants unique to an individual strain (private SNPs and indels), genotypes for all variant sites identified in each of the 13 strains were computationally derived for all strains in the MGP variation catalogue (36 strains). Private SNPs were then identified as high quality homozygous alternative sites, in which the same site must be a high quality homozygous reference allele in at least 30 other strains. A maximum of five strains were allowed a missing genotype or low quality call at the same site to account for the genetic diversity of wild-derived strains in the MGP variation catalogue. This resulted in $2.33 \mathrm{M}(8.52 \%)$ private SNPs across the 13 strains, although $1.82 \mathrm{M}$ of these private sites were identified in the wild-derived MOLF/EiJ strain (Table 1). This accounted for $9.56 \%$ of all MOLF/EiJ SNP sites, which was much higher than the proportion of private variation identified for any of the other 12 strains (0.18-1.6 \%) (Table 1). DBA/1J had the lowest proportion of private SNPs due to the genetic similarity of DBA/1J and DBA/2J [34]; $90.72 \%$ of DBA/1J SNPs are shared with DBA/2J.

Similar to the total variation per strain, most private SNPs are located in intergenic (45.67-57.68\% per strain) and intronic regions (13.28-23.32\%) while 7.18-10.3\% per strain are within $5 \mathrm{~kb}$ of a coding gene. In total, 7237 private missense SNPs were identified in 4615 genes across all 13 strains although 4086 of these are estimated to have more than one consequence (Additional file 6). Most private missense SNPs are predicted to be 
tolerated by SIFT or have a GMS $<100$. Using SIFT predictions, 2146 SNPs affecting 1768 genes were predicted to be deleterious. Similarly, using GMS predictions, only 893 SNPs affecting 818 genes were predicted to cause moderate changes and 461 SNPs affecting 446 genes were predicted to cause radical changes to the CDS. As expected, only a small number of private variants led to changes in stop codons (93 stop gains and 12 stop losses) or were predicted to affect splice acceptor or donor sites (46 and 53 SNPs, respectively) although many of these SNPs were predicted to have more than one consequence (51 stop gains, 8 stop losses, 43 splice acceptor and 43 splice donor sites).

Ninety-one genes are affected by a private stop gain. For example, a $\mathrm{C} \rightarrow \mathrm{T}$ transition at amino acid position 208 of the bone morphogenetic protein 5 (BMP5) gene causes a stop gain private to the SEA/GnJ strain. Bone morphogenetic proteins are signalling molecules involved in the development of bone and cartilage tissue [35]. This strain is known to be homozygous for this mutation (also known as the short ear allele). Mice homozygous for the short ear allele exhibit abnormal skeletal development leading to smaller and weaker bones, reduced shape and size of the external ear, a reduced number of ribs and alterations in size and shape of many small bone elements [36].

Nearly half of private indels $(48.96 \%)$ are found in intergenic regions, $18.61 \%$ are intronic and $8.89 \%$ are within $5 \mathrm{kbp}$ of a coding gene while almost one-quarter $(23.27 \%)$ of indels are predicted to have two or more consequences. Only $84(0.015 \%)$ private frameshift mutations were identified, and 61 of these were identified in MOLF/EiJ. As expected, very little private variation was observed in the C57BL/10J strain compared to the C57BL/6J reference genome. These two strains were separated in 1921 from the same C57BL progenitor colony and no other strains are known to have contributed to the parentage of either strain [3]. Eighty-three genes were identified that carry a private frameshift mutation in one of the 13 strains such as a ' $\mathrm{T}$ ' insertion resulting in a frameshift at amino acid position 431 of the phosphorylase kinase alpha 1 (Phka1) gene. This mutation, which is private to the I/LnJ strain, causes a premature stop codon in the downstream coding region resulting in a great reduction of Phkal transcript in skeletal muscle and partial reduction in heart and brain [37]. Additionally, this mutation has been implicated in myopathy and glycogen storage disease in both humans and mice [38].

\section{Large structural deletions and insertions}

A total of 79,943 large ( $>100 \mathrm{bp}$ ) deletions and 143,854 insertions were identified across all of the strains (Table 1). Of the large deletions identified, 20,122 were novel and a large proportion $(17,514)$ of these were private to one of the 13 strains when compared to the full variation catalogue of 36 strains. Structural variant insertions were also identified for all strains in the MGP variation catalogue, including the 13 strains from this study. A total of 143,854 insertions were identified across the 13 strains examined in this study. Approximately $48.34 \%$ of these were novel $(69,542), 63,262$ of which were private to one of the 13 strains when compared to the MGP variation catalogue.

To assess the accuracy of our calling pipeline, polymerase chain reaction (PCR) validation deletions and insertions were obtained for seven MGP strains [39]. Structural variants were identified in these seven strains using the same approach as for the 13 strains in this study. Sensitivity estimates were estimated for each of the seven strains (Table S2 of Additional file 1). All sensitivity estimates for each strain were between 92.05-94.12\% for deletions and 81.94-94.25\% for insertions, which are comparable to estimates previously published for MGP strains [40, 41].

Although none of the 13 strains included in this study were among the seven strains, 151 PCR-validated deletions and 84 insertions were available for the DBA/2J strain. The 151 PCR-validated deletions identified in the DBA/2J strain [39] were compared with the deletion call set identified in DBA/1J. Although these are two separate strains, they are genetically identical at many loci throughout the genome. Most of the 151 deletions overlapped with a deletion identified in DBA/1J from our analysis (131). This meant that 20 deletions PCRvalidated in DBA/2J were not identified in the DBA/1J strain. Upon visual inspection of these deletions using the alignment data and the integrative genomics viewer [42], eight were identified as real differences between the strains. When true structural variant differences between the strains are removed from the sensitivity analysis, the sensitivity increases to $91.61 \%(131 / 143)$ which is similar to the proportion of SNP sites that are identical between the strains (90.72\%).

Sensitivity estimates were also calculated for SV insertions identified in the DBA/1J strain using PCR-validated calls identified in the DBA/2J strain. Most DBA/2J insertions were also identified in DBA/1J (76/84). Similar to deletions, sensitivity ( $90.48 \%$ ) of the SV insertion calling pipeline was similar to estimates reported previously in MGP strains [40, 41].

Little is known about structural variation in the genomes of the 13 strains included in this study. However, many of the structural variants which we identified were also shared with another strain in the MGP variation catalogue. For example, a 6-kb deletion in BFSP2 which results in alterations of optical lens quality has previously been identified in the FVB/NJ and 129 strains [43]. 
From our analysis, we identified this mutation in both the NZB/B1NJ and NZW/LacJ strains. Although neither of these strains are known models for examining optical lens quality, utilising either strain in studies which may rely on vision or lens quality could lead to spurious results.

Coding sequences for all genes annotated to the mouse genome were then compared against our deletion call set to identify genes which may have their function disrupted. A total of 4954 structural deletions overlapped the coding sequence of 3316 genes. Of these, 2462 were novel and 2110 were private to one of the 13 strains in our study. Similarly, 4620 structural insertions overlapped the coding sequence of 5076 genes, many of which were either novel (2557) or private (2366) to one of the 13 strains.

\section{$\mathrm{RF} / \mathrm{J}$ strain enriched for private missense variants in cancer susceptibility genes}

To investigate the potential biological mechanisms underlying disease phenotypes for which these strains are used to study, two analyses were carried out. The first study focused on the RF/J strain, which was developed at the Rockefeller Institute through breeding of the A, R and S strains [44]. Due to the natural predisposition of RF/J mice to develop myeloid leukaemia, thymic lymphoma and reticulum cell sarcomas [44], this strain is an important model used to study cancer onset, progression and pathogenesis. This strain also has a propensity to develop lung, ovary and hematopoietic tumours. In particular, this strain is widely used to study the effects of radiation-induced myeloid leukaemia due to its high induction rate (between 50-90 \%) [44].

To identify and prioritise candidate genes for further analysis, only genes which contained at least one missense variant private to the $\mathrm{RF} / \mathrm{J}$ strain were considered for further analysis. A total of 122 genes were identified which met this condition (Additional file 7). Several of the genes in this list, such as Adam15 [45], Cdh1 [46], Msh3 and Kit [47], are known candidates for roles in tumorigenesis and cancer pathogenesis. For example, mice nullizygous for the $M s h 3$ gene are reported to have a significantly higher spontaneous incidence rate of tumours than wild-type counterparts [48]. The Msh3 gene is involved in the DNA repair mechanism, and plays an important role in mismatch recognition [49]. The mismatch repair pathway identifies and eliminates errors that arise during DNA replication and defects in this mechanism can lead to the accumulation of mutations and cancer [50]. In fact, loss or silencing of the Msh3 gene has been reported to frequently occur in several human cancers including lung [51] and breast [52]. In addition, associations of mutations in the Msh3 gene to colon [53] and prostate [54] cancer in humans have been reported.

Another gene involved in the DNA damage response, $P A L B 2$, was also identified in our candidate set. Mutations of this gene are known to increase risk of several cancers including breast [55] and pancreatic cancer [56]. Mutations of $P A L B 2$ are commonly identified in patients with Fanconi anaemia. In fact, patients with PALB2-related Fanconi anaemia also have an increased predisposition to malignancies associated with acute myeloid leukaemia [57] and Wilms tumours [58]. Interestingly, the WT1 (Wilms tumour) gene was also identified in our candidate gene set as carrying a private missense SNP.

Additional genes of particular note in our candidate set included the tumour suppressor gene $C d h 1$, mutations in which are known to predispose to a wide range of cancer types [59], and the Fas gene. Fas, a member of the tumour necrosis factor (TNF) receptor family of proteins, plays a key role in activation of apoptosis and has been implicated in the pathogenesis of several malignancies [60]. Dysregulation of apoptosis-related pathways is also commonly observed in cancer and defects of this signalling process are known to contribute to drug therapy insensitive tumours [61]. Furthermore, polymorphisms in the promoter of the Fas gene have been associated with an increased risk of developing acute myeloid leukaemia in humans [62]. Interestingly, following $\gamma$-irradiation-induced thymic lymphoma, Fas expression has also been shown to be lower in the RF/J strain compared to the SPRET/EiJ and BALB/cJ strains, both of which are known to be more resistant to this induced phenotype [63]. Although no private missense SNPs in this gene were identified in the BALB/cJ strain, one missense SNP in exon 2 was identified. Similarly, 17 missense SNPs were identified in the wild-derived SPRET/EiJ strain, 14 of which were private to this strain. Due to each of the abovementioned strains containing missense SNPs in this gene, it is difficult to determine the exact role this gene or mutations in this gene may play in the pathogenesis of cancer phenotypes observed in the RF/J strain.

To examine the overall effect of private missense mutations in RF/J, we identified biological pathways overrepresented in this gene set. Significantly over-represented REACTOME [64] and KEGG [65] pathways were identified using the hypergeometric test available through the pathway over-representation analysis tools at InnateDB [66]. After correcting $p$ values using the Benjamini-Hochberg method, four pathways were significantly overrepresented $(p<0.05)$. Notably, this list included the KEGG pathway 'Pathways in cancer' $(p=0.046)$. Each of the remaining three significantly over-represented pathways were related to extracellular matrix (ECM) functions (Table 3). In fact, the most significantly over-represented 
Table 3 Significantly over-represented biological pathways and candidate genes identified in RF/J

\begin{tabular}{llll}
\hline Pathway name & Database & $p$ value (corrected) & Candidate genes \\
\hline Degradation of the extracellular matrix & REACTOME & $4.91 \times 10^{-3}$ & Adam15, Cdh1, Col4a4, Eln, Mmp11 \\
Extracellular matrix organization & REACTOME & $1.15 \times 10^{-2}$ & Adam15, Cdh1, Col4a4, Eln, Lama3, Mmp11 \\
ECM-receptor interaction & KEGG & $1.32 \times 10^{-2}$ & Col4a4, Itga10, Itga7, Lama3 \\
Pathways in cancer & KEGG & $4.56 \times 10^{-2}$ & Cdh1, Col4a4, Fas, Kit, Lama3, Msh3 \\
\hline
\end{tabular}

Candidate genes are those that are found in the respective pathways and contained a missense SNP private to the RF/J strain. Corrected $p$ values are based on the Benjamini-Hochberg method

REACTOME and KEGG pathways were 'Degradation of the extracellular matrix' $(p=0.0049)$ and 'ECM-receptor interaction' ( $p=0.013)$, respectively.

The ECM is a fundamental component of the cellular microenvironment, composed of a complex mix of structural and functional molecules [67]. The ECM has important roles in a diverse set of functions including cellular development, tissue differentiation, growth and tissue homeostasis [68]. In addition, components of the ECM are in continual contact with epithelial cells and mediate signalling of processes involved in regulation of cell proliferation, apoptosis and differentiation [69]. Notably, 'ECM-receptor interaction' has previously been found to be enriched for genes differentially regulated in cancer cell lines compared to normal tissue [70] and in tumour expression patterns compared to normal tissue [71]. In addition, abnormal ECM has been implicated in the progression of cancer. In fact, altered ECM is commonly found in patients with cancer [72].

To investigate whether these results may be due to an annotation bias within KEGG or REACTOME, the same pathway over-representation analysis was performed for each of the 13 strains' private missense mutations (Additional file 8). No strain's gene set was enriched for genes involved in 'Pathways in cancer,' 'Degradation of the extracellular matrix' or 'Extracellular matrix organization'. However, 'ECM-receptor interaction' was also significantly over-represented in the MOLF/EiJ gene set $(p=0.0026)$. However, it may not be appropriate to carry out such analysis using wild-derived strains such as MOLF/EiJ due to the relatively low representation of wild-derived strains in the MPG catalogue compared to the classical inbred strains. For example, approximately 56 times as many private SNPs were identified in MOLF/EiJ compared to $\mathrm{RF} / \mathrm{J}$ (Table 1 ). Consequently, there are nearly 28 times as many genes which met the inclusion criteria using MOLF/EiJ (3366 genes) when compared to the RF/J gene set (122 genes).

\section{CIA candidate genes and biological pathways}

The second study carried out focused on the genetic and phenotypic differences between the genetically similar DBA/1J and DBA/2J strains. The DBA strain was developed by Clarence C. Little in 1909 and is the oldest of all inbred mouse strains. During 1929 and 1930, crosses between substrains led to the establishment of several new substrains including DBA/1J and DBA/2J [34]. Just over $90.72 \%$ of SNP sites identified in the DBA/1J strain are identical to the DBA/2J strain; however, these strains exhibit several phenotypic differences including total body weight and body fat weight [73]. In addition, differences in circulating plasma levels of triglycerides, high-density lipoprotein, leptin and insulin have been reported between DBA/1J and DBA/2J [34]. Notably, these strains also display divergent susceptibility to CIA, a model for human RA. The DBA/1J strain, sequenced as part of this study, is susceptible to CIA whereas the DBA/2J strain is resistant [74]. To identify potential variation, and genes that may be involved in these divergent phenotypes, all SNPs identified in DBA/ 1J which are shared with the DBA/2J strain were removed. This meant we were left with a set of DBA/ 1J-specific SNPs. However, despite the genetic similarity of these strains, they have different haplotypes at the MHC locus [75]. To remove SNPs in this region not associated with CIA susceptibility, all SNPs shared with the CIA resistant but $\mathrm{MHC}$ identical $\mathrm{FVB} / \mathrm{NJ}$ strain were also removed. A total of 258,416 SNPs remained in this DBA/1J-specific CIA susceptibility candidate set. From this, all genes containing at least a single missense variant with the additional SIFT annotation of 'deleterious' were identified (123 SNPs identified in 101 genes). Similar to the previous study, this gene set was then used to identify significantly over-represented biological pathways using the tools available from InnateDB [66].

Murine CIA, a widely used model for human RA, is characterised as an autoimmune inflammatory joint disease which primarily affects synovial joints ultimately leading to joint impairment and disability [14]. The pathogenesis of RA involves the influx of both innate and adaptive immune cells, which in turn promote pro-inflammatory cytokine production and decreased synthesis of anti-inflammatory cytokines [76]. Normally the inflammatory response is balanced by the release of anti-inflammatory molecules which prevent chronic inflammation. Disruption of the anti-inflammatory balancing mechanism can lead to sustained chronic inflammation as observed in RA. Interestingly, our list of candidate 
genes containing a missense deleterious SNP also included the anti-inflammatory mediator interleukin-10 receptor a subunit (IL10RA). Interleukin-10 (IL10) is an antiinflammatory cytokine whose functions are mediated by binding to IL1ORA which in turn stimulates signalling cascades used to inhibit production of pro-inflammatory cytokines such as TNF- $\alpha$ [77]. $I L-10^{-/}$DBA/1J mice have increased CIA severity compared to mice either homozygous or heterozygous at the same locus [78]. Similarly, therapeutic treatment with $I L-10$ has been shown to suppress the development of CIA in rats [79]. In addition, mutations in the ILIORA have been implicated in the pathogenesis of several inflammatory autoimmune diseases such as murine lupus [80] and ulcerative colitis [81]. Diminished IL1O signalling, through mutations in the IL10RA, have been implicated in the severity of several autoimmune diseases including inflammatory bowel disease and arthritis [81, 82].

Similar to RF/J, pathway over-representation analysis was carried out using the candidate gene set identified in the DBA/1J analysis. A total of 10 pathways were significantly over-represented (corrected $p<0.05$ ), many of which are related to innate immunity and inflammation signalling (Table 4). The most significantly overrepresented pathway was 'cellular adhesion molecules (CAMs)' $\left(p=3.98 \times 10^{-4}\right)$. CAMs are expressed on the surface of cells and play a critical role in the inflammatory response. This pathway has been shown to be enriched for genes with a differentially methylated signature in synovial tissue of RA patients compared to patients without RA [83]. Several additional KEGG pathways identified in our analysis have also been implicated in the pathogenesis of RA including 'cytokine-cytokine receptor interaction' ( $p=0.047)$ and 'antigen processing and presentation' ( $p=0.011)$ [84]. Notably, none of the candidate genes identified in the cytokine-cytokine receptor interaction pathway are shared with candidate genes identified in either CAMs or antigen processing and presentation pathways, whereas all candidates identified in the antigen processing and presentation pathway are also found in the CAMs pathway. Given the complex nature of RA, it is not surprising that many genes and biological pathways contribute to the pathogenesis of RA [85]. Complimenting evidence from individual candidate gene lists with biological information available through interaction and pathway databases may help identify new targets for therapeutic treatments of complex diseases such as RA. In spite of this, additional work utilising other high-throughput technologies (e.g. RNA-seq and proteomics) and in vivo models is still required to fully understand the onset and severity of CIA and ultimately RA.

\section{Conclusions}

We report the significant expansion of the MGP resource to incorporate deep whole-genome sequencing of 36 inbred laboratory strains. The amount of SNP variation found has increased from $56 \mathrm{M}$ to $71 \mathrm{M}$ sites, comparable in genetic variation to the final 1000 Genomes set [86]. In this paper, we focus on the 13 new laboratory strains that include further representatives of the classical laboratory and wild-derived strains. The original project sought to sequence as widely possible from the laboratory mouse subspecies groups. These 13 new strains mean that we now have several examples of highly related pairs of strains that are known to display divergent phenotypes, which could accelerate the identification of causative variants.

We focus on two specific examples of how the variation data detected can be used to prioritise genes of interest for well-known phenotypes in the new strains. In the first example, we focus on the RF/J strain which has a highly penetrant predisposition to develop acute myeloid leukaemia, thymic lymphoma and reticulum cell

Table 4 Significantly over-represented biological pathways and candidate genes identified in DBA/1 J

\begin{tabular}{llll}
\hline Pathway name & Database & p value (corrected) & Candidate genes \\
\hline Cell adhesion molecules (CAMs) & KEGG & $3.98 \times 10^{-4}$ & Esam, H2-M10, H2-M10, H2-T22, Itga9, Sdc3 \\
Type I diabetes mellitus & KEGG & $7.54 \times 10^{-3}$ & H2-M10, H2-M10, H2-T22 \\
Graft-versus-host disease & KEGG & $7.59 \times 10^{-3}$ & H2-M10, H2-M10, H2-T22 \\
Autoimmune thyroid disease & KEGG & $8.55 \times 10^{-3}$ & H2-M10, H2-M10, H2-T22 \\
Allograft rejection & KEGG & $1.01 \times 10^{-2}$ & H2-M10, H2-M10, H2-T22 \\
Antigen processing and presentation & KEGG & $1.06 \times 10^{-2}$ & H2-M10, H2-M10, H2-T22 \\
Viral myocarditis & KEGG & $1.14 \times 10^{-2}$ & H2-M10, H2-M10, H2-T22 \\
Endocytosis & KEGG & $3.07 \times 10^{-2}$ & Arap3, H2-M10, H2-M10, H2-T22 \\
Linoleic acid metabolism & KEGG & $4.49 \times 10^{-2}$ & Cyp2C44, Cyp2C50 \\
Cytokine-cytokine receptor interaction & KEGG & $4.73 \times 10^{-2}$ & II1Ora, II28ra, Tnfrsflb, Tnfrsf8
\end{tabular}

Candidate genes are genes that are found in the over-represented pathways and which contained a missense, deleterious SNP found in DBA/1J and not in DBA/2J or FVB/NJ. Corrected $p$ values were calculated using the Benjamini-Hochberg method 
sarcomas. We used the expanded variation catalogue to identify candidate genes containing missense mutations that are private to the RF/J strain. Several notable genes with a known role in cancer were identified in our candidate gene set (e.g. Msh3, Kit, Fas). This gene set was then further interrogated leading to the identification of biological pathways with potential roles in cancer susceptibility in this strain. In the second analysis, we investigate the genetic basis of collagen induced arthritis (a model of human rheumatoid arthritis) using two genetically very similar strains with divergent phenotypes of susceptible (DBA/1J) and resistant (DBA/2J). Through comparison of variants identified in both strains, we were able to identify a candidate gene set of 101 genes involved in the susceptibility of DBA/1J to this induced phenotype. Further analysis of this gene set revealed several biological processes involved in immune signalling and inflammation which may play a role in the pathogenesis of human RA. When using the mutation catalogues for genetic mapping studies, it must be noted that the structural variation calls have an error rate of up to $10 \%$. A recent study found that more accurate structural variation calls can be derived from long read technologies compared to those produced by short read detection methods [87], therefore we expect that future releases of variants on these strains will be even more accurate.

These strains represent a significant increase in the disease models available in the MGP variation catalogue which will enable improved dissection of the genetic variation underlying many human diseases. The expansion of the catalogue of sequenced strains means that we can identify mutations that are more likely to be truly private to individual strains, thereby reducing the size of candidate variant sets. Sequencing of genetically diverse strains, such as the wild-derived MOLF/EiJ, are invaluable resources for identifying new genetic variation not present in classical inbred laboratory strains. However, in wild-derived strains many loci are composed of novel alleles and divergent haplotypes from the current reference genome [88]. Efforts are currently ongoing to create de novo assembled genomes for several classical and wild-derived strains [89] which will enable more accurate examination of the genomic structure and genetic variation responsible for phenotypes observed in these strains.

\section{Methods}

\section{DNA sequencing and read alignment}

DNA from a single male of each inbred mouse strain was obtained from the Jackson Laboratories. Jackson Laboratory stock numbers for each strain are contained in Table S1 of Additional file 1. DNA was sheared to 200-300 bp fragment size using a Covaris S2. A single
Illumina TruSeq v3 sequencing library was created for each strain according to manufacturer's protocols. Each library was sequenced on a HiSeq 2000 over four lanes. Each lane was genotype checked using Samtools/ Bcftools v1.2 (http://www.htslib.org) 'gtcheck' command by comparing variant sites against the mouse Hapmap [22]. Library and sequencing statistics for each sample are available in Table S3 of Additional file 1.

Sequencing reads from each lane were aligned to the C57BL/6 J GRCm38 (mm10) mouse reference genome [5] using BWA-MEM (v0.7.5) [90] with default parameters. For each library, aligned reads from each lane were merged using Picard Tools (v1.64) [91], resulting in a single BAM file corresponding to all aligned reads for a single strain. Each BAM file (one for each strain) was then sorted and filtered for possible PCR and optical duplicates using Picard Tools (v1.64). To improve SNP and indel calling, the GATK v3.0 'IndelRealigner' tool [92], was used to realign reads around indels using default options.

\section{SNP and indel discovery}

SNPs and indels were identified using a combination of SAMtools mpileup (v1.1) [93] and BCFtools call (v1.1). SAMtools mpileup captures summary information from input BAMs and uses alignment information to estimate genotype likelihoods. BCFtools, based on the VCFtools package [94], then uses this information, with the inclusion of a prior, to perform the variant calling. The following options were specified for SAMtools; 't

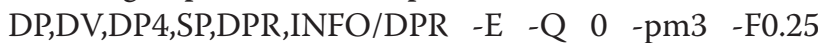
-d500' and BCFtools call; '-mv -f GQ,GP -p 0.99'. To improve accuracy of indel calls, indels were then leftaligned and normalised using the bcftools norm function with the parameters '-D - $-\mathrm{m}+$ indels'.

To ensure high quality variants were only used for later analysis, bcftools annotate was used to soft filter SNPs and indels identified. These filters are used to identify low quality variant calls and remove false SNP and indel calls due to alignment artifacts. Only high quality (i.e. passed all filters) homozygote variants were retained. Filter values and cutoffs used to identify low quality variants are described in Table S4 of Additional file 1 .

\section{SNP and indel annotation and prediction of consequences}

Using mouse gene models obtained from Ensembl 78, the Ensembl VEP tool (version 78) [23] was used to predict the effect of SNPs and indels on gene transcripts. VEP assigns variants to functionally relevant categories based on the predicted effect that a variant has on a transcript or gene. Consequences assigned to variants within coding regions of a gene include stop losses/gains, 
missense variants and splice variants. Notably, a single variant may have different estimated consequences between alternative transcripts of the same gene. To predict whether amino acid substitutions would be damaging, GMS [29] were calculated for each variant. GMS are used to predict the effect of substitutions between amino acids. Variants with a calculated GMS between 100 and 150 $(100<$ GMS $\leq 150)$ were considered moderately radical and variants with a GMS $>150$ were considered radical changes. Additionally, SIFT [95] scores were estimated for all variants. SIFT predicts amino acid substitution effects on protein function and classifies variants as being either tolerated or deleterious.

\section{Structural variation calling}

A combination of BreakDancer [96], $\mathrm{cnD}$ [97] and LUMPY [98] were used to identify larger structural deletions/losses (greater than $100 \mathrm{bp}$ ). BreakDancer identifies large deletions using deviations from the expected mate pair distance and $\mathrm{cnD}$ identifies losses of genomic segments using changes in mapping depth across regions. LUMPY utilises multiple SV signals, including read-pair and split-read information to identify potential SV breakpoints. All parameters used by BreakDancer, $\mathrm{cnD}$ and LUMPY are described in Table S5 of Additional file 1. The deletion call sets for each strain were then collapsed into strain-specific nonredundant sets of deletions by merging deletions with a reciprocal overlap greater than $90 \%$. This meant that for each merged deletion there were two possible deletion breakpoints, the primary breakpoints, which are those identified by BreakDancer, $\mathrm{cnD}$ or LUMPY, and a secondary breakpoint, which are the outer bounds of merged deletions.

To identify SV insertions, RetroSeq (transposable element insertions) [99], cnD (copy number gains) [97], scalpel [100] and manta (both novel sequence insertions) [101] were used. Parameters for each analysis are contained in Table S5 of Additional file 1.

Calls made by each tool were then filtered to remove any SV within $500 \mathrm{bp}$ of an assembly gap or $20 \mathrm{~kb}$ of a telomere or centromere as mapping artifacts in these regions can cause false SV calls. Additionally, SVs greater than $1 \mathrm{MB}$ in size were removed as these were generally false positive calls due to poor mapping among reads.

\section{Identification and count of private variation}

Variants identified in the 13 strains examined in this study were then incorporated in the mouse genomes variation catalogue. The MGP variation catalogue now contains variants identified in 36 different inbred laboratory mouse strains. To obtain a list of high confidence variants private to each strain, sequencing data from the remaining 23 strains contained in the MGP variation catalogue was aligned to the mouse reference genome using the same pipeline as described above.

Similarly, the same SAMtools and BCFtools pipeline as above was used to identify SNPs and short indels. SNP and indel locations from all 36 strains were collated into a single file which was used to generate genotypes at those locations in all strains using samtools mpileup with the -l option enabled (i.e. report the genotype at all locations listed). The same bcftools call parameters as the initial variant calling step were used. This allowed the identification of high quality homozygous reference locations, as well as low quality regions and regions with no sequence information (uncallable regions).

Private SNPs were identified as locations in which a single high quality homozygote alternative variant was called in only one of the 36 strains. In the remaining 35 strains, the location must be a high quality homozygous reference genotype allowing a maximum of five low quality or missing genotypes in any of the strains. Similarly, private indels were identified for each strain. A private indel was defined as any high quality homozygote alternative indel unique to a single strain.

To identify SV deletions that may be the result of the same ancestral event, deletions were first identified in each of the remaining 23 MGP strains using the same approach as described above. Deletions identified in each individual strain were compared with the deletions identified in all other strains (13 strains sequenced as part of this study and the additional 23 MGP strains). A non-redundant across-strain deletion set was identified, as before, using a greater than $90 \%$ reciprocal overlap to merge deletions. Following this merge, private SV deletions were identified as those that did not overlap with another deletion, and thus were not merged with any other deletion.

Similarly, as with predicted SV deletions, SV insertions were identified for all 36 strains contained in the MGP variation catalogue, including the 13 strains from this study. The insertion call sets for each strain were then merged and used to identify insertions private to each strain.

\section{Variants calls accuracy}

To assess the accuracy of SNP calls made for each strain, genotypes were obtained from the mouse HapMap resource [22] for each of our strains and compared with the corresponding SNP identified in our catalogue for each of the 13 strains separately. The mouse HapMap contains approximately 138,000 homozygous sites genotyped in 94 inbred laboratory mouse strains. Genotype locations for each of the 13 strains were mapped from $\mathrm{mm} 9$ to $\mathrm{mm} 10$ of the reference genome, and any missing genotypes were removed from further analysis. Between 115,572 (MOLF/EiJ) and 129,825 (C58/J) 
remained after mapping SNP locations from $\mathrm{mm} 9$ to $\mathrm{mm} 10$ and removing missing genotypes. Sensitivity of the SNP calling pipeline was estimated by calculating the number of genotypes concordant between the HapMap and the variation catalogue for each of the 13 strains. The number of genotypes from the HapMap available for comparison with each of the 13 strains and the respective concordance rate are contained in Additional file 4.

A dataset of PCR validated SV insertions and deletions for seven MGP strains (AKR/J, A/J, BALB/cJ, DBA/2J, $\mathrm{CBA} / \mathrm{J}, \mathrm{C} 3 \mathrm{H} / \mathrm{HeJ}$ and $\mathrm{LP} / \mathrm{J}$ ) were obtained from Yalcin et al. [39]. Coordinates for all variants were first mapped from NCBIm37 to GRCm38. Using the SV calling pipeline described above, sequencing data for these seven strains was used to call structural variants that could be compared to the PCR-validated set to assess the accuracy of our SV calling pipeline. In addition, SVs identified in the DBA/1J from our study were compared to the PCR-validated variants for DBA/2J (Table S2 of Additional file 1).

\section{Identification of candidate genes through prioritisation of damaging SNPs}

To demonstrate the usefulness of this data, two studies investigating the genetic basis of cancer susceptibility and CIA were carried out. The first investigation involved identifying all genes that contained a missense variant that was private to the RF/J strain. This strain is an important model used to investigate the onset and progression of several cancers including leukaemia and reticulum cell sarcomas. This gene set was then used to identify any biological pathways that may be enriched for genes from this set (described below).

The second investigation was carried out to investigate the genetic basis of CIA. Two strains, DBA/1J and DBA/ $2 J$, were chosen as they are genetically similar $(>90.72 \%$ of DBA/1J SNPs are shared with DBA/2J) but phenotypically divergent for susceptibility to CIA; DBA/1J is susceptible, however DBA/2J is resistant. All SNPs identified in these two strains were collated into a single file. The SNPs specific to DBA/2J or shared with DBA/2J were then removed leaving a DBA/1J-specific set of SNPs. However, these strains differ at the MHC locus. To account for the differing MHC alleles, SNPs shared with the CIA-resistant $\mathrm{FVB} / \mathrm{NJ}$ strain were also removed. This SNP set was then filtered to only include missense SNPs with a SIFT annotation of deleterious. All genes containing these variants were then identified, and this gene set was used to identify biological pathways that may be involved in CIA susceptibility.

\section{Pathway over-representation analysis}

For each of the candidate gene sets described above, a pathway over-representation analysis was performed to identify biological pathways or processes that are statistically enriched for this gene set. This was done using the pathway over-representation tools available from InnateDB [66] and analysed pathway annotations available from the REACTOME [64] and KEGG [65] databases. This test was based on the hypergeometric algorithm and correction of $p$ values was done using the BenjaminiHochberg method. Significantly over-represented pathways were defined as those with a corrected $p<0.05$.

\section{Additional files}

\section{Additional file 1: Supplemental tables. Supplemental tables S1-S5. (PDF 109 kb)}

Additional file 2: SNP, indel and SV density plots for all strains. SNP, indel and SV (deletions and insertions) density plots for all chromosomes (1-19, $X$ and $Y$ ) for each strain. (PDF $3421 \mathrm{~kb}$ )

Additional file 3: Pairwise variant comparisons. The number of SNPS and indels shared between any two strains contained in the MGP variation catalogue. (XLSX 24 kb)

Additional file 4: Per strain genotype concordance with HapMap. Total HapMap positions available for comparison with each strain and the corresponding number of concordant sites. (XLSX $4 \mathrm{~kb}$ )

Additional file 5: VEP, GMS and SIFT annotations for SNPS and indels in each of the 13 strains. Total number of SNPs and indels annotated into each functional consequence predicted by VEP. Total number of SNPS with an estimated moderate (using GMS), radical (GMS), tolerated (using SIFT) and deleterious (SIFT) effect is also provided. (XLSX 8 kb)

Additional file 6: Private SNPS with multiple VEP consequences. Private SNPs with multiple predicted VEP consequences for each strain strain. Consequence predictions are based on gene models obtained from Ensembl 78. (TXT 66669 kb)

Additional file 7: Candidate genes identified in the RF/J analysis. All genes which contained at least one missense SNP only found in the RF/J strain. (XLSX 8 kb)

Additional file 8: Per strain over-represented pathways using genes containing private missense SNPS. For each strain separately significantly over-represented pathways were identified using only the genes which contained at least one private missense variant. (XLSX $56 \mathrm{~kb}$ )

\begin{abstract}
Abbreviations
AML, acute myeloid leukaemia; bp, base pair; CDS, coding sequence; CIA, collagen-induced arthritis; GATK, Genome Analysis ToolKit; GMS, Grantham Matrix Scores; Indel, insertion/deletion polymorphism; MGP, Mouse Genomes Project; PCR, polymerase chain reaction; SNP, single nucleotide polymorphism; SV, structural variant; UTR, untranslated region
\end{abstract}

\section{Acknowledgements}

Not applicable.

\section{Funding}

This work was supported by the Medical Research Council (MR/L007428/1), BBSRC (BB/M000281/1) and the Wellcome Trust. DJA is supported by Cancer Research UK and the Wellcome Trust. This research was supported in part by the Intramural Research Program of the NIH, National Cancer Institute, Center for Cancer Research.

\section{Availability of data and materials}

All raw sequencing reads are available from the European Nucleotide Archive (ERP000927; see Table S1 in Additional file 1 for sample identifiers). The sequence variants are available from the European Variation Archive (PRJEB11471) and the Mouse Genomes Project ftp site (ftp://ftp-mouse.sanger.ac.uk). 


\section{Authors' contributions}

KWH, JF, DJA and TMK initiated the ideas for the study. AJD, KW and TMK carried out the computational analysis and drafted the manuscript. All authors read and approved the manuscript.

\section{Competing interests}

The authors declare that they have no competing interests.

\section{Ethics approval and consent to participate}

DNA provided by The Jackson Laboratories was obtained in accordance with local ethical guidelines and regulations.

\section{Author details}

${ }^{1}$ Wellcome Trust Sanger Institute, Hinxton, Cambridge CB10 1HH, UK. ${ }^{2}$ The Wellcome Trust Centre for Human Genetics, Roosevelt Drive, Oxford OX3 7BN, UK. ${ }^{3}$ Laboratory of Cancer Biology and Genetics, NCl, NIH, Bethesda, Maryland, USA.

\section{Received: 8 February 2016 Accepted: 12 July 2016 Published online: 01 August 2016}

\section{References}

1. Paigen K. One hundred years of mouse genetics: an intellectual history. II. The molecular revolution (1981-2002). Genetics. 2003;163:1227-35.

2. Michaud EJ, Culiat CT, Klebig ML, Barker PE, Cain KT, Carpenter DJ, et al. Efficient gene-driven germ-line point mutagenesis of C57BL/6 J mice. BMC Genomics. 2005:6:164.

3. Beck JA, Lloyd S, Hafezparast M, Lennon-Pierce M, Eppig JT, Festing MF, et al. Genealogies of mouse inbred strains. Nat Genet. 2000;24:23-5.

4. Mouse Genome Sequencing Consortium, Waterston RH, Lindblad-Toh K Birney E, Rogers J, Abril JF, et al. Initial sequencing and comparative analysis of the mouse genome. Nature. 2002;420:520-62.

5. Church DM, Goodstadt L, Hillier LW, Zody MC, Goldstein S, She X, et al. Lineage-specific biology revealed by a finished genome assembly of the mouse. PLoS Biol. 2009;7, e1000112.

6. van der Weyden L, Adams DJ, Bradley A. Tools for targeted manipulation of the mouse genome. Physiol Genomics. 2002;11:133-64.

7. Skarnes WC, Rosen B, West AP, Koutsourakis M, Bushell W, lyer V, et al. A conditional knockout resource for the genome-wide study of mouse gene function. Nature. 2011:474:337-42.

8. Frazer KA, Eskin E, Kang HM, Bogue MA, Hinds DA, Beilharz EJ, et al. A sequence-based variation map of 8.27 million SNPs in inbred mouse strains. Nature. 2007:448:1050-3.

9. Keane TM, Goodstadt L, Danecek P, White MA, Wong K, Yalcin B, et al. Mouse genomic variation and its effect on phenotypes and gene regulation. Nature. 2011:477:289-94.

10. Furth J, Seibold H, Rathbone R. Experimental studies on lymphomatosis of mice. Am J Cancer. 1933;19:521-604.

11. Cole R, Furth J. Experimental studies on the genetics of spontaneous leukemia in mice. Cancer Res. 1941;1:957-65.

12. Kitahama K, Valatx $J \mathrm{~L}$, Jouvet M. Paradoxical sleep deprivation and performance of an active avoidance task: impairment of c57BR mice and no effect in C57BL/6 mice. Physiol Behav. 1981;27:41-50.

13. Bovet $D$, Bovet-Nitti F, Oliverio A. Effects of nicotine on avoidance conditioning of inbred strains of mice. Psychopharmacologia. 1966;10:1-5.

14. Brand DD, Latham KA, Rosloniec EF. Collagen-induced arthritis. Nat Protoc. 2007;2:1269-75.

15. Singh RR, Ebling FM, Albuquerque DA, Saxena V, Kumar V, Giannini EH, et al. Induction of autoantibody production is limited in nonautoimmune mice. J Immunol. 2002;169:587-94.

16. Gude WD, Lupton AC. Spontaneous glomerulosclerosis in aging RF mice. J Gerontol. 1960;15:373-6.

17. Johnson KR, Zheng QY, Weston MD, Ptacek $\sqcup$, Noben-Trauth K. The Mass1frings mutation underlies early onset hearing impairment in BUB/BnJ mice, a model for the auditory pathology of Usher syndrome IIC. Genomics. 2005;85:582-90.

18. Paul LK. Developmental malformation of the corpus callosum: a review of typical callosal development and examples of developmental disorders with callosal involvement. J Neurodev Disord. 2011;3:3-27.

19. Green MC. Mechanism of the pleiotropic effects of the short-ear mutant gene in the mouse. J Exp Zool. 1968;167:129-50.

20. Chang B, Hawes NL, Hurd RE, Davisson MT, Nusinowitz S, Heckenlively JR. Retinal degeneration mutants in the mouse. Vision Res. 2002:42:517-25.
21. Kane KL, Longo-Guess CM, Gagnon LH, Ding D, Salvi RJ, Johnson KR. Genetic background effects on age-related hearing loss associated with Cdh23 variants in mice. Hear Res. 2012;283:80-8.

22. Kirby A, Kang HM, Wade CM, Cotsapas C, Kostem E, Han B, et al. Fine mapping in 94 inbred mouse strains using a high-density haplotype resource. Genetics. 2010;185:1081-95.

23. McLaren W, Pritchard B, Rios D, Chen Y, Flicek P, Cunningham F. Deriving the consequences of genomic variants with the Ensembl API and SNP Effect Predictor. Bioinformatics. 2010;26:2069-70.

24. Pan $\mathrm{H}-J$, Agate DS, King BL, Wu MK, Roderick SL, Leiter EH, et al. A polymorphism in New Zealand inbred mouse strains that inactivates phosphatidylcholine transfer protein. FEBS Lett. 2006;580:5953-8.

25. Wu MK, Cohen DE. Altered hepatic cholesterol metabolism compensates for disruption of phosphatidylcholine transfer protein in mice. Am J Physiol Gastrointest Liver Physiol. 2005;289:G456-61.

26. Wu MK, Hyogo H, Yadav SK, Novikoff PM, Cohen DE. Impaired response of biliary lipid secretion to a lithogenic diet in phosphatidylcholine transfer protein-deficient mice. J Lipid Res. 2005;46:422-31.

27. Pittler SJ, Baehr W. Identification of a nonsense mutation in the rod photoreceptor CGMP phosphodiesterase beta-subunit gene of the rd mouse. Proc Natl Acad Sci U S A. 1991;88:8322-6.

28. Ng PC, Henikoff S. SIFT: Predicting amino acid changes that affect protein function. Nucleic Acids Res. 2003:31:3812-4.

29. Grantham R. Amino acid difference formula to help explain protein evolution. Science. 1974;185:862-4.

30. Ichihara M, Hara T, Takagi M, Cho LC, Gorman DM, Miyajima A. Impaired interleukin-3 (IL-3) response of the A/J mouse is caused by a branch point deletion in the IL-3 receptor alpha subunit gene. EMBO J. 1995;14:939-50.

31. Wetsel RA, Fleischer DT, Haviland DL. Deficiency of the murine fifth complement component (C5). A 2-base pair gene deletion in a 5'-exon. J Biol Chem. 1990;265:2435-40.

32. Nilsson UR, Müller-Eberhard HJ. Deficiency of the fifth component of complement in mice with an inherited complement defect. J Exp Med. 1967;125:1-16.

33. Howell GR, Soto I, Ryan M, Graham LC, Smith RS, John SWM. Deficiency of complement component 5 ameliorates glaucoma in DBA/2 J mice. J Neuroinflammation. 2013:10:76.

34. Stylianou IM, Langley SR, Walsh K, Chen Y, Revenu C, Paigen B. Differences in DBA/1 J and DBA/2 J reveal lipid OTL genes. J Lipid Res. 2008:49:2402-13.

35. Tsumaki N, Nakase T, Miyaji T, Kakiuchi M, Kimura T, Ochi T, et al. Bone morphogenetic protein signals are required for cartilage formation and differently regulate joint development during skeletogenesis. J Bone Miner Res. 2002;17:898-906.

36. Kingsley DM, Bland AE, Grubber JM, Marker PC, Russell LB, Copeland NG, et al. The mouse short ear skeletal morphogenesis locus is associated with defects in a bone morphogenetic member of the TGF beta superfamily. Cell. 1992;71:399-410.

37. Schneider A, Davidson JJ, Wüllrich A, Kilimann MW. Phosphorylase kinase deficiency in I-strain mice is associated with a frameshift mutation in the alpha subunit muscle isoform. Nat Genet. 1993;5:381-5.

38. Wuyts W, Reyniers E, Ceuterick C, Storm K, de Barsy T, Martin J-J. Myopathy and phosphorylase kinase deficiency caused by a mutation in the PHKA1 gene. Am J Med Genet A. 2005;133A:82-4.

39. Yalcin B, Wong K, Bhomra A, Goodson M, Keane TM, Adams DJ, et al. The fine-scale architecture of structural variants in 17 mouse genomes. Genome Biol. 2012;13:R18.

40. Yalcin B, Wong K, Agam A, Goodson M, Keane TM, Gan X, et al. Sequencebased characterization of structural variation in the mouse genome. Nature. 2011;477:326-9.

41. Wong K, Bumpstead S, Van Der Weyden L, Reinholdt LG, Wilming LG, Adams DJ, et al. Sequencing and characterization of the FVB/NJ mouse genome. Genome Biol. 2012;13:R72.

42. Robinson JT, Thorvaldsdóttir H, Winckler W, Guttman M, Lander ES, Getz G, et al. Integrative genomics viewer. Nat Biotechnol. 2011;29:24-6.

43. Simirskii VN, Lee RS, Wawrousek EF, Duncan MK. Inbred FVB/N mice are mutant at the cp49/Bfsp2 locus and lack beaded filament proteins in the lens. Invest Ophthalmol Vis Sci. 2006:47:4931-4.

44. Wolman SR, MCMorrow LE, Cohen MW. Animal model of human disease: myelogenous leukemia in the RF mouse. Am J Pathol. 1982;107:280-4.

45. Najy AJ, Day KC, Day ML. ADAM15 supports prostate cancer metastasis by modulating tumor cell-endothelial cell interaction. Cancer Res. 2008; 68:1092-9. 
46. Richards FM, McKee SA, Rajpar MH, Cole TR, Evans DG, Jankowski JA, et al. Germline E-cadherin gene (CDH1) mutations predispose to familial gastric cancer and colorectal cancer. Hum Mol Genet. 1999:8:607-10.

47. Care RS, Valk PJM, Goodeve AC, Abu-Duhier FM, Geertsma-Kleinekoort WMC, Wilson GA, et al. Incidence and prognosis of c-KIT and FLT3 mutations in core binding factor (CBF) acute myeloid leukaemias. $\mathrm{Br} J$ Haematol. 2003;121:775-7

48. Hegan DC, Narayanan L, Jirik FR, Edelmann W, Liskay RM, Glazer PM. Differing patterns of genetic instability in mice deficient in the mismatch repair genes Pms2, Mlh1, Msh2, Msh3 and Msh6. Carcinogenesis. 2006;27:2402-8.

49. van Oers JMM, Edwards Y, Chahwan R, Zhang W, Smith C, Pechuan X, et al. The MutS $\beta$ complex is a modulator of p53-driven tumorigenesis through its functions in both DNA double-strand break repair and mismatch repair. Oncogene. 2014;33:3939-46.

50. Peltomäki P. Deficient DNA, mismatch repair: a common etiologic factor for colon cancer. Hum Mol Genet. 2001;10:735-40.

51. Benachenhou N, Guiral S, Gorska-Flipot I, Labuda D, Sinnett D. High resolution deletion mapping reveals frequent allelic losses at the DNA mismatch repair loci hMLH1 and hMSH3 in non-small cell lung cancer. Int J Cancer. 1998;77: 173-80.

52. Benachenhou N, Guiral S, Gorska-Flipot I, Labuda D, Sinnett D. Frequent loss of heterozygosity at the DNA mismatch-repair loci hMLH1 and hMSH3 in sporadic breast cancer. Br J Cancer. 1999;79:1012-7.

53. Orimo H, Nakajima E, Yamamoto M, Ikejima M, Emi M, Shimada T. Association between single nucleotide polymorphisms in the hMSH3 gene and sporadic colon cancer with microsatellite instability. J Hum Genet. 2000;45:228-30.

54. Hirata H, Hinoda Y, Kawamoto K, Kikuno N, Suehiro Y, Okayama N, et al. Mismatch repair gene MSH3 polymorphism is associated with the risk of sporadic prostate cancer. J Urol. 2008;179:2020-4.

55. Antoniou AC, Casadei S, Heikkinen T, Barrowdale D, Pylkäs K, Roberts J, et al. Breast-cancer risk in families with mutations in PALB2. N Engl J Med. 2014; 371:497-506.

56. Salo-Mullen EE, O'Reilly EM, Kelsen DP, Ashraf AM, Lowery MA, Yu KH, et al. Identification of germline genetic mutations in patients with pancreatic cancer. Cancer. 2015;121:4382-8.

57. Auerbach AD, Allen RG. Leukemia and preleukemia in Fanconi anemia patients. A review of the literature and report of the International Fanconi Anemia Registry. Cancer Genet Cytogenet. 1991;51:1-12.

58. Tischkowitz M, Xia B. PALB2/FANCN - recombining cancer and Fanconi anemia. Cancer Res. 2010;70:7353-9.

59. Hansford S, Kaurah P, Li-Chang H, Woo M, Senz J, Pinheiro H, et al. Hereditary diffuse gastric cancer syndrome: $\mathrm{CDH} 1$ mutations and beyond. JAMA Oncol. 2015:1:23-32.

60. Park WS, Oh RR, Kim YS, Park JY, Lee SH, Shin MS, et al. Somatic mutations in the death domain of the Fas (Apo-1/CD95) gene in gastric cancer. J Pathol. 2001;193:162-8

61. Debatin K-M. Apoptosis pathways in cancer and cancer therapy. Cancer Immunol Immunother. 2004;53:153-9.

62. Sibley K, Rollinson S, Allan JM, Smith AG, Law GR, Roddam PL, et al. Functional FAS promoter polymorphisms are associated with increased risk of acute myeloid leukemia. Cancer Res. 2003;63:4327-30.

63. Villa-Morales M, Santos J, Fernández-Piqueras J. Functional Fas (Cd95/Apo-1) promoter polymorphisms in inbred mouse strains exhibiting different susceptibility to gamma-radiation-induced thymic lymphoma. Oncogene. 2006;25:2022-9.

64. Joshi-Tope G, Gillespie M, Vastrik I, D'Eustachio P, Schmidt E, de Bono B, et al. Reactome: a knowledgebase of biological pathways. Nucleic Acids Res. 2005; 33:D428-32.

65. Kanehisa M, Goto S. KEGG: kyoto encyclopedia of genes and genomes. Nucleic Acids Res. 2000;28:27-30.

66. Breuer K, Foroushani AK, Laird MR, Chen C, Sribnaia A, Lo R, et al. InnateDB: systems biology of innate immunity and beyond-recent updates and continuing curation. Nucleic Acids Res. 2013;41:D1228-33.

67. Hynes RO. The extracellular matrix: not just pretty fibrils. Science. 2009; 326:1216-9

68. Mouw JK, Ou G, Weaver VM. Extracellular matrix assembly: a multiscale deconstruction. Nat Rev Mol Cell Biol. 2014;15:771-85.

69. Lu P, Takai K, Weaver VM, Werb Z. Extracellular matrix degradation and remodeling in development and disease. Cold Spring Harb Perspect Biol. 2011;3. http://www.ncbi.nlm.nih.gov/pmc/articles/PMC3225943/. Accessed 4 Feb 2016.
70. Ertel A, Verghese A, Byers SW, Ochs M, Tozeren A. Pathway-specific differences between tumor cell lines and normal and tumor tissue cells. Mol Cancer. 2006;5:55.

71. Krupp M, Maass T, Marquardt JU, Staib F, Bauer T, König R, et al. The functional cancer map: a systems-level synopsis of genetic deregulation in cancer. BMC Med Genomics. 2011:4:53.

72. Cox TR, Erler JT. Remodeling and homeostasis of the extracellular matrix: implications for fibrotic diseases and cancer. Dis Model Mech. 2011;4:165-78.

73. Svenson KL, Von Smith R, Magnani PA, Suetin HR, Paigen B, Naggert JK, et al. Multiple trait measurements in 43 inbred mouse strains capture the phenotypic diversity characteristic of human populations. J Appl Physiol. 2007;102:2369-78.

74. Donate PB, Fornari TA, Junta CM, Magalhães DA, Macedo C, Cunha TM, et al. Collagen induced arthritis (CIA) in mice features regulatory transcriptional network connecting major histocompatibility complex $(\mathrm{MHC} \mathrm{H2)} \mathrm{with}$ autoantigen genes in the thymus. Immunobiology. 2011;216:591-603.

75. Campfield BT, Nolder CL, Davis A, Hirsch R, Nowalk AJ. The DBA/1 strain is a novel mouse model for experimental Borrelia burgdorferi infection. Clin Vaccine Immunol. 2012;19:1567-73.

76. Heruth DP, Gibson M, Grigoryev DN, Zhang LQ, Ye SQ. RNA-seq analysis of synovial fibroblasts brings new insights into rheumatoid arthritis. Cell Biosci. 2012;2:43.

77. Riley JK, Takeda K, Akira S, Schreiber RD. Interleukin-10 receptor signaling through the JAK-STAT pathway. Requirement for two distinct receptor-derived signals for anti-inflammatory action. J Biol Chem. 1999;274:16513-21.

78. Finnegan A, Kaplan CD, Cao Y, Eibel H, Glant TT, Zhang J. Collagen-induced arthritis is exacerbated in IL-10-deficient mice. Arthritis Res Ther. 2003;5:R18-24.

79. Persson S, Mikulowska A, Narula S, O'Garra A, Holmdahl R. Interleukin-10 suppresses the development of collagen type II-induced arthritis and ameliorates sustained arthritis in rats. Scand J Immunol. 1996:44:607-14.

80. Qi Z-M, Wang J, Sun Z-R, Ma F-M, Zhang Q-R, Hirose S, et al. Polymorphism of the mouse gene for the interleukin 10 receptor alpha chain (II10ra) and its association with the autoimmune phenotype. Immunogenetics. 2005;57: 697-702.

81. Moran CJ, Walters TD, Guo C-H, Kugathasan S, Klein C, Turner D, et al. IL-10R polymorphisms are associated with very-early-onset ulcerative colitis. Inflamm Bowel Dis. 2013;19:115-23.

82. Hermann J, Gruber S, Neufeld JB, Grundtner P, Graninger M, Graninger WB, et al. IL10R1 loss-of-function alleles in rheumatoid arthritis and systemic lupus erythematosus. Clin Exp Rheumatol. 2009;27:603-8.

83. Whitaker JW, Shoemaker R, Boyle DL, Hillman J, Anderson D, Wang W, et al. An imprinted rheumatoid arthritis methylome signature reflects pathogenic phenotype. Genome Med. 2013;5:40.

84. Whitaker JW, Boyle DL, Bartok B, Ball ST, Gay S, Wang W, et al. Integrative omics analysis of rheumatoid arthritis identifies non-obvious therapeutic targets. PLoS One. 2015;10, e0124254.

85. Glocker MO, Guthke R, Kekow J, Thiesen H-J. Rheumatoid arthritis, a complex multifactorial disease: on the way toward individualized medicine. Med Res Rev. 2006;26:63-87.

86. 1000 Genomes Project Consortium, Auton A, Brooks LD, Durbin RM, Garrison EP, Kang HM, et al. A global reference for human genetic variation. Nature. 2015;526:68-74.

87. Gordon D, Huddleston J, Chaisson MJP, Hill CM, Kronenberg ZN, Munson KM, et al. Long-read sequence assembly of the gorilla genome. Science. 2016;352: aae0344.

88. Lilue J, Müller UB, Steinfeldt T, Howard JC. Reciprocal virulence and resistance polymorphism in the relationship between Toxoplasma gondii and the house mouse. eLife. 2013;2, e01298.

89. Adams DJ, Doran AG, Lilue J, Keane TM. The Mouse Genomes Project: a repository of inbred laboratory mouse strain genomes. Mamm Genome. 2015;26:403-12.

90. Li H. Aligning sequence reads, clone sequences and assembly contigs with BWA-MEM. http://arxiv.org/abs/1303.3997.

91. The Picard toolkit. http://broadinstitute.github.io/picard/.

92. McKenna A, Hanna M, Banks E, Sivachenko A, Cibulskis K, Kernytsky A, et al. The Genome Analysis Toolkit: a MapReduce framework for analyzing next-generation DNA sequencing data. Genome Res. 2010;20:1297-303.

93. Li H, Handsaker B, Wysoker A, Fennell T, Ruan J, Homer N, et al. The Sequence Alignment/Map format and SAMtools. Bioinformatics. 2009;25:2078-9.

94. Danecek P, Auton A, Abecasis G, Albers CA, Banks E, DePristo MA, et al. The variant call format and VCFtools. Bioinformatics. 2011;27:2156-8. 
95. Kumar P, Henikoff S, Ng PC. Predicting the effects of coding non-synonymous variants on protein function using the SIFT algorithm. Nat Protoc. 2009:4:1073-81.

96. Chen K, Wallis JW, McLellan MD, Larson DE, Kalicki JM, Pohl CS, et al. BreakDancer: an algorithm for high-resolution mapping of genomic structural variation. Nat Methods. 2009;6:677-81.

97. Simpson JT, McIntyre RE, Adams DJ, Durbin R. Copy number variant detection in inbred strains from short read sequence data. Bioinformatics. 2010;26:565-7.

98. Layer RM, Chiang C, Quinlan AR, Hall IM. LUMPY: a probabilistic framework for structural variant discovery. Genome Biol. 2014;15:R84.

99. Keane TM, Wong K, Adams DJ. RetroSeq: transposable element discovery from next-generation sequencing data. Bioinformatics. 2013;29:389-90.

100. Narzisi G, O'Rawe JA, lossifov I, Fang H, Lee Y-H, Wang Z, et al. Accurate de novo and transmitted indel detection in exome-capture data using microassembly. Nat Methods. 2014:11:1033-6.

101. Chen X, Schulz-Trieglaff O, Shaw R, Barnes B, Schlesinger F, Källberg M, et al. Manta: rapid detection of structural variants and indels for germline and cancer sequencing applications. Bioinformatics. 2016;32:1220-2.

Submit your next manuscript to BioMed Central and we will help you at every step:

- We accept pre-submission inquiries

- Our selector tool helps you to find the most relevant journal

- We provide round the clock customer support

- Convenient online submission

- Thorough peer review

- Inclusion in PubMed and all major indexing services

- Maximum visibility for your research

Submit your manuscript at www.biomedcentral.com/submit
Biomed Central 